\title{
Dust emission from inhomogeneous interstellar clouds: Radiative transfer in 3D with transiently heated particles
}

\author{
M. Juvela ${ }^{1}$ and P. Padoan ${ }^{2}$ \\ 1 Helsinki University Observatory, Tähtitorninmäki, PO Box 14, 00014 University of Helsinki, Finland \\ 2 Jet Propulsion Laboratory, 4800 Oak Grove Drive, MS 169-506, California Institute of Technology, USA
}

Received 16 July 2002 / Accepted 30 September 2002

\begin{abstract}
Due to the complexity of their structure, the theoretical study of interstellar clouds must be based on threedimensional models. It is already possible to estimate the distribution of equilibrium dust temperature in fairly large 3D models and, therefore, also to predict the resulting far-infrared and sub-mm emission. Transiently heated particles introduce, however, a significant complication and direct calculation of emission at wavelengths below $100 \mu \mathrm{m}$ is currently not possible in 3D models consisting of millions of cells. Nevertheless, the radiative transfer problem can be solved with some approximations. We present a numerical code for continuum radiative transfer that is based on the idea of a "library" describing the relation between the intensity of the local radiation field and the resulting dust emission spectrum. Given this mapping it is sufficient to simulate the radiation field at only a couple of reference wavelengths. Based on the library and local intensities at the reference wavelengths, the radiative transfer equation can be integrated through the source and an approximation of the emission spectrum is obtained. Tests with small models for which the radiative transfer problem can be solved directly show that with our method, one can easily obtain an accuracy of a few per cent. This depends, however, on the opacity of the source and the type of the radiation sources included. As examples we show spectra computed from three-dimensional MHD simulations containing up to $128^{3}$ cells. The models represent starless, inhomogeneous interstellar clouds embedded in the normal interstellar radiation field. The intensity ratios between IRAS bands show large variations that follow the filamentary structure of the density distribution. The power law index of the spatial power spectrum of the column density map is -2.8 . In infrared maps temperature variations increase the power at high spatial frequencies, and in a model with average visual extinction $\left\langle A_{\mathrm{V}}\right\rangle \sim 10$ the power law index varies between -2.5 and -2.7 . Assuming constant dust properties throughout the cloud, the IRAS ratio $\left\langle I_{60} / I_{100}\right\rangle$ decreases in densest cores only by a factor of $\sim 4$ compared with the value in diffuse medium. Observations have shown that in reality the ratio can decrease twice as much even in optically thinner clouds. This requires that most of the small grains are removed in these regions, and possibly a modification of the properties of large grains.
\end{abstract}

Key words. ISM: clouds - infrared: ISM - radiative transfer

\section{Introduction}

Present knowledge on the large scale infrared emission from interstellar dust is based largely on the all-sky surveys performed by the IRAS and the COBE satellites. IRAS was the first to observe the sky at a resolution of a few arc minutes at wavelengths between 12 and $100 \mu \mathrm{m}$. Clear variations were observed in the infrared spectrum of interstellar clouds, e.g. the ratio of $60 \mu \mathrm{m}$ vs. $100 \mu \mathrm{m}$ dropping towards dense clouds (e.g. Laureijs et al. 1996; Abergel et al. 1994). These were interpreted as the result of abundance variations between classical, large grains and very small dust particles that are transiently heated above the equilibrium temperature (e.g. Désert et al. 1990). Emission in the mid-infrared and especially the IRAS

Send offprint requests to: $\mathrm{M}$. Juvela, e-mail: mjuvela@astro.helsinki.fi
$12 \mu \mathrm{m}$ band, are now believed to be caused by even smaller grains or very large molecules, the most common candidates being Polycyclic Aromatic Hydrocarbons (PAHs) (Léger et al. 1989; Désert et al. 1990; Li \& Draine 2001). The PAH emission traces the warmer outer surfaces of the clouds.

The COBE/DIRBE instrument covered a wider wavelength range from near-infrared up to $240 \mu \mathrm{m}$ and the FIRAS instrument up to $1 \mathrm{~cm}$. The spatial resolution was, however, comparatively poor i.e. $\sim 40^{\prime}$ for DIRBE and $\sim 7^{\circ}$ for FIRAS. Nevertheless, the extended wavelength coverage revealed surprisingly large quantities of cold dust that were not observed with IRAS. Detailed analysis of the COBE data has shown that at least two emission components are needed to explain the observed far-infrared spectra. For most of the diffuse regions the dust emission follows a modified black body law with temperature $T \sim 17.5 \mathrm{~K}$ (Boulanger et al. 1996; 
Dwek et al. 1997). Lagache et al. (1998) found, however, a colder component $(T \sim 15 \mathrm{~K})$ associated with molecular clouds. Finkbeiner et al. (1999) reached similar conclusions but included an even colder, $T \sim 9 \mathrm{~K}$, component.

Recent observations with the PRONAOS balloon borne observatory have confirmed the lowering of the colour temperature towards molecular clouds and, e.g. in the case of the Polaris flare $\left(A_{\mathrm{V}} \sim 1\right)$, towards moderately dense regions (Stepnik et al. 2001). Similar effects have been seen in ISOCAM studies towards cirrus like clouds $\left(A_{\mathrm{V}} \sim 0.5\right.$; Miville-Deschênes et al. 2002) where the temperature variations caused by extinction are quite insignificant. The observed increase in the sub-mm emission can be explained by grain growth and the formation of large dust aggregates (Stepnik et al. 2001; Cambrésy et al. 2001) and in dense regions the very small grains seem to have disappeared almost completely. Conversely, in the more diffuse medium, large quantities of small grains can be produced by grain shattering (see e.g. Miville-Deschênes et al. 2002).

Based on these observations and theoretical studies we have obtained a rough picture of the dust properties and infrared emission from interstellar clouds. In the outer layers we have the PAH emission and the warm, very small dust grains. Deeper in the clouds the extinction of short wavelength radiation reduces mid-infrared emission. At the same time, the grains start to form larger aggregates and/or acquire mantles as gas molecules freeze onto them. This increases the far-infrared and sub-mm emissivity relative to the absorption at short wavelengths and the physical grain temperatures also drop. The colour temperature therefore drops to approximately $10 \mathrm{~K}$ (or possibly even lower) in dense cloud cores (e.g. Juvela et al. 2002).

Theoretical and laboratory studies have provided us with some information on the properties of likely dust grain materials. Together with radiative transfer calculations, these have made it possible to determine size distributions for the different dust components consistent with the emission properties (e.g. Désert et al. 1990; Li \& Draine 2001). On the other hand, radiative transfer models can be used to study the variations of these properties in individual objects. These previous examples show that the dust emission spectrum is variable over the whole range from near-infrared to sub-mm. The changes are related to the local radiation field and the local density. So far, modelling has concentrated either on the determination of the dust emission under given radiation conditions, or at most in one-dimensional, spherical models divided into some tens of cells.

In the related field of the modelling of molecular line emission, three-dimensional models have already been used for some time (Park \& Hong 1995; Park et al. 1996; Juvela 1998; Padoan et al. 1998; Juvela et al. 2001). In that field, inhomogeneous source structure has an even stronger effect on the emerging radiation and, on the other hand, direct calculations are already possible for models with up to $\sim 100^{3}$ cells or, with approximate methods, even higher (Ossenkopf 2002).

The continuum emission is not as directly linked with the local, physical conditions. It is, nevertheless, affected by the variations in the radiation field (extinction of the external radiation field and internal sources) and the changes in the dust properties. So far dust emission has not been studied with general three-dimensional cloud models. The main reason is the complexity caused by the transiently heated small dust particles. In order to be able to predict the emission accurately, the distribution of the grain temperatures must be first determined in each computational cell. Each dust population must be discretized into separate grain size intervals. For each of these, a couple of hundred enthalpy bins are needed and the solution of the associated set of linear equations takes typically several seconds. This becomes significant when the model contains millions of cells and, in practice, it limits direct calculations to one- or possibly two-dimensional models.

We will discuss how this limitation can be overcome by suitable approximations without siginificantly affecting the accuracy of the results. This will be based on the assumption that the local spectral energy distribution can be deduced from the intensity at suitably selected reference wavelengths. One must first determine a mapping between these intensities and the resulting local spectral energy distribution. The radiative transfer problem needs only to be solved at the reference wavelengths, and by using the established mapping, one can solve the radiative transfer problem. The solution is approximative but can be made sufficiently accurate within the limitations of present day computers. The method makes it possible to study explicitly the effects that the cloud structure has on the infrared emission, and the method could be useful e.g. in the simulation of the spectral energy distribution of galaxies.

We first explain the implementation of the radiative transfer program (Sect. 2) and discuss its accuracy (Sect. 3). In Sect. 4 we apply the method to three-dimensional model clouds that are based on numerical simulations of supersonic magnetohydrodynamic (MHD) turbulence and we show some observable consequences of the inhomogeneity of the clouds. Finally, in Sect. 4.3 we discuss some qualitative effects of spatial variations in the dust properties.

\section{The program}

\subsection{Direct solution}

The full radiative transfer problem can be divided into two largely independent parts. In the first one determines the intensity of the radiation field at each position of the cloud (in our case in each discrete cell of finite volume). In the second part this information and the selected dust model are used to derive the local dust temperature and emission. In interstellar clouds dust emission mainly takes place in the infrared part of the spectrum. As long as the external radiation field is relatively weak the dust emission has only a small effect on the heating of grains (see e.g. Hollenbach et al. 1991; Bernard et al. 1992). This is true for all models considered in this paper and, therefore, the two parts of the computation mentioned above can be carried out sequentially without iteration. In the following we will ignore other processes affecting the dust temperature. For example, the gas-dust coupling becomes important only at densities above $\gtrsim 10^{5} \mathrm{~cm}^{-3}$ (e.g. Krügel \& Walmsley 1984) and the clouds considered here have much lower densities. 
Our three-dimensional model clouds are divided into cubic cells, each with a constant density. First, the optical depths for absorption and scattering as well as the parameters of the scattering function are calculated for each cell. The usual HenyeyGreenstein formula (1941) and the asymmetry parameter $g$ are used to define the scattering function. The radiation field is calculated with Monte Carlo methods. Model photons are initiated at random locations at the cloud boundary in order to simulate the background radiation, and within the cloud to simulate emission from the dust and possible internal sources. The path of the model photon is tracked in the cloud. A model photon represents a number of real photons. Absorbed photons are counted in each cell that is crossed, and from time to time, the model photon is scattered towards a new direction as determined by the dust model. Because of the frequency dependence of the scattering probability, the simulation has to be carried out separately for each frequency.

The simulation determines the spectrum of the incoming radiation for each cell. In the second phase this is used to compute the dust temperature. The dust model consists of several dust populations, and each population is further discretized according to grain size. For each of these we have a probability distribution of the grain enthalpy, discretized usually to a few hundred bins. The transition probabilities for cooling processes are calculated beforehand, based on the specific heat and absorption cross sections. The transitions upwards in energy are obtained by integration from the simulated intensity spectrum. The formulae we use are exact only for a probability distribution that is flat a function of grain energy (temperature). We have also implemented the "thermal discrete" method described by Draine \& Li (2001) and have tested that our method gives, with the used discretizations, identical results for the studied wavelength range. Once the number of grains as the function of temperature (enthalpy) is known the local emission can be calculated.

The two steps still need to be iterated, if the dust emission itself contributes to the grain heating. This requires, however, either the presence of strong, local heating sources or very high extinction. Bernard et al. (1992) studied the case of spherical clouds heated by normal ISRF (a slightly modified version of the ISRF given by Mathis et al. 1983). The IR heating was found to be insignificant for visual extinctions $A_{\mathrm{V}}<100^{\mathrm{m}}$ through the cloud, and even with $A_{\mathrm{V}}=1000^{\mathrm{m}}$, it increased the sub-mm emission only by $\sim 5 \%$. The effect is due to an increase in the temperature of the large, classical grains. Shorter wavelength emission is in practice not affected, since the infrared photons emitted by the dust grains are not energetic enough to cause significant transient heating of the smaller grains.

\subsection{The library method}

For large, three-dimensional models consisting of $>100^{3}$ cells the described direct approach is no longer possible. Simulation of the necessary number of frequencies $(>100)$ could still be done, but it would possibly take days on a workstation, depending on the required accuracy. The main problem is the solution of the linear equations. Each dust population is discretized into ten or more size intervals, each further divided into possibly several hundred enthalpy intervals. The solution of the associated linear equations for the transition probabilities requires more than a second per cell, or a month for a model with $100^{3}$ computational cells. The direct approach is clearly limited to very small models (or very large supercomputers).

The spectrum of the local radiation field is very similar in many parts of the cloud. For example, in a homogeneous, semiinfinite plane or a homogeneous sphere, the intensity and spectrum of incoming radiation depends only on one parameter, the distance from the free surface. In these cases one could calculate the dust emission at certain depths and obtain the emission from intermediate positions with interpolation.

Inside an inhomogeneous or an irregularly shaped cloud there is more variation in the spectral energy distribution of the incoming radiation. Usually, in a homogeneous cloud, the extinction reduces radiation more strongly at short wavelengths. This is not true, however, in the extreme case of inpenetrable clumps. If clumps absorb all incoming radiation irrespective of its wavelength, the intensity inside the cloud will be reduced proportionally to the solid angle blocked by the clumps, while the shape of the spectrum will remain unchanged. Clearly, in a general case, the changes in the radiation field cannot be tracked with just one parameter (e.g. visual extinction). We argue, however, that for a given model, the spectrum can be sufficiently constrained with knowledge of the intensity at possibly only two or three reference wavelengths, $\lambda_{i}^{\text {ref }}$.

The first step in our approximation is to find the range of intensities and spectral energy distributions of the incoming radiation. For this purpose, we reduce the discretization of the model down to $\sim 32^{3}$ cells. This allows fast solution of the radiative transfer part of the problem. The result is the simulated intensity in each cell. We take the range of intensities found at the first reference wavelength, $\lambda_{1}^{\text {ref }}$, and discretize that into logarithmic intensity intervals. The procedure is repeated recursively at the other reference wavelengths. The result is a tree structure that represents a discretization of all observed intensity combinations, $\left(I\left(\lambda_{1}^{\text {ref }}\right), I\left(\lambda_{2}^{\text {ref }}\right), \ldots\right)$. In the leaves of the tree we store the average, full spectrum that corresponds to a unique combination of intensities at the reference wavelengths. If the reference wavelengths are suitably selected, the tree and knowledge of intensities at reference wavelengths can be used to obtain an estimate of the full spectrum.

The second step of our approximation is the solution of the dust emission corresponding to each spectrum of incoming radiation that was stored in the leaves of the tree. This way, we obtain a mapping from the incoming intensity at the reference wavelengths to the corresponding full spectrum of local dust emission. We call this structure "the library" (see Fig. 1).

To predict dust emission from a large, three-dimensional model, we first simulate the radiation field at the small number of reference wavelengths, $\lambda_{i}^{\text {ref }}$. The radiative transfer equation can then be directly integrated through the cloud, using in each cell the library to transform the simulated values of incoming intensity at the reference wavelengths into the full spectrum of emitted intensity. We call this "the library method". When we look up the emission spectrum from the library, we also perform linear interpolation based on the values $I\left(\lambda_{i}^{\text {ref }}\right)$. Again, 


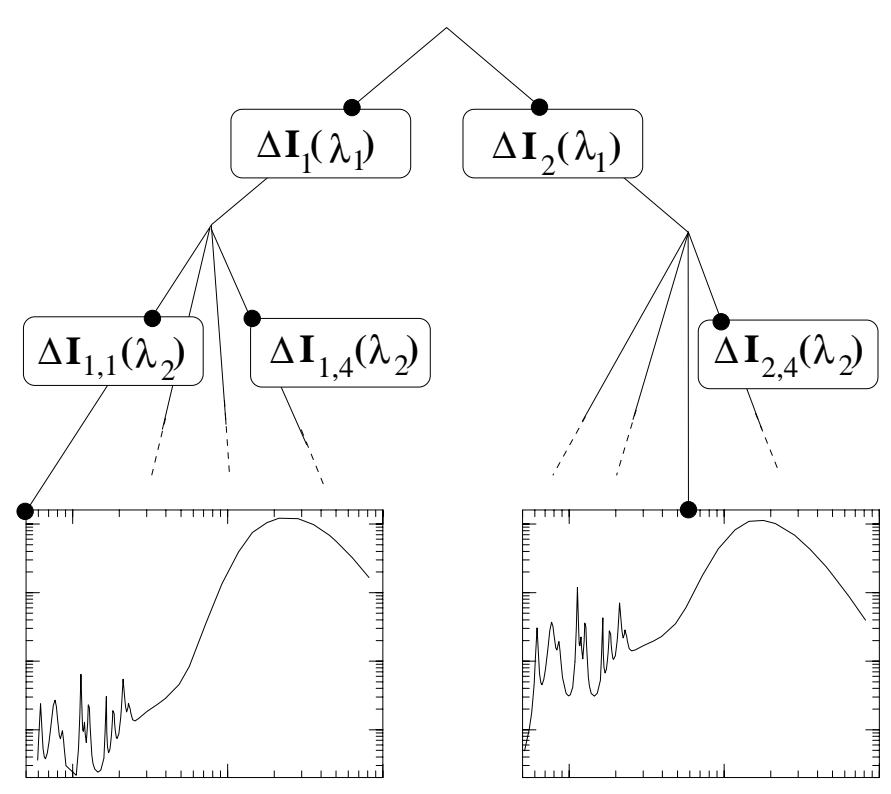

Fig. 1. A schematic view of the tree structure used in the library method. At each level the intensity of the incoming radiation at one wavelength is examined and each subnode corresponds to a different intensity interval, $\Delta I$. The leaves of the tree correspond to a unique combination of intensity values at the reference wavelengths and they hold the corresponding emission spectrum. Once the intensity of the incoming radiation is known at reference wavelengths, the emission can be read from the tree.

if absorbed dust emission is important (e.g. close to strong sources or in very opaque regions) iteration is needed.

\section{Tests of the library method}

\subsection{The model clouds and the dust model}

We will now estimate the accuracy of the approximate method described in Sect. 2.2. The method is based on the discretization of the incoming intensity into bins for which the corresponding dust emission spectrum is only computed for the average spectrum. The accuracy can therefore always be improved by selecting a finer discretization and/or including more reference wavelengths. In order to obtain a significant advantage over the direct solution, the total number of spectrum bins must, however, be much less than the number of cells in the model.

In this paper, we limit the study to starless clouds heated by the interstellar radiation field. We take as a test case an MHD simulation which is the model $C$ from Juvela et al. (2001). The model is both supersonic and super-Alfvénic with acoustic and Alfvénic Mach numbers $M \approx M_{\mathrm{A}} \approx 10$. The MHD simulation included self-gravity.

The dust model is taken from Li \& Draine (2001) and includes silicate grains (sizes $a>3.5 \AA$ ), graphite grains $(a \gtrsim 50 \AA$ ) and PAHs (from $a=3.5 \AA$ to $\sim 50 \AA$ ). The PAH properties correspond to cold neutral medium. The grain size distributions are the same as in Li \& Draine (2001). The only difference is the way $\mathrm{PAH}$ and graphite grain populations are joined. Instead of a gradual change in the description of absorption coefficient (Li \& Draine 2001, Eq. (2)) we only join
Table 1. Parameters of the model clouds used in the testing: name of the model, average density assuming linear size $L=6 \mathrm{pc}$, and the average and maximum values of visual extinction seen towards the direction for which the dust emission was computed.

\begin{tabular}{crrr}
\hline \hline model name & $\begin{array}{r}\langle n\rangle \\
{\left[\mathrm{cm}^{-3}\right]}\end{array}$ & $\left\langle A_{\mathrm{V}}\right\rangle$ & $A_{\mathrm{V}}^{\mathrm{MAX}}$ \\
\hline $\mathrm{C} 0$ & 320 & 2.76 & 24.6 \\
$\mathrm{C} 1$ & 1280 & 11.04 & 98.5 \\
$\mathrm{C} 2$ & 5120 & 44.16 & 394.0 \\
\hline
\end{tabular}

the grain size distributions smoothly around $40 \mu \mathrm{m}$. The optical properties are taken from the files available through the website of B. Draine. The original references are Laor \& Draine (1993) for graphite and silicate grains and Li \& Draine (2001) for the PAH properties.

At this point, we are interested in the differences between the two methods (i.e. direct solution and the library method) and not in the absolute accuracy. Therefore, the number of grain size intervals $(\sim 10)$, grain energies $(<300)$ and simulated frequencies $(\sim 100)$ were kept to a minimum that still reproduces the Li \& Draine (2001) spectra for diffuse ISM within $\sim 10 \%$ accuracy over the studied wavelength range, 5$300 \mu \mathrm{m}$. The largest differences result from a different method used to join the PAH and graphite grain populations around grain size $40 \mu \mathrm{m}$. The interstellar radiation field adopted is from Mathis et al. (1983).

The original cloud model represented a cloud with a linear size $\sim 6 \mathrm{pc}$ and a mean density of $320 \mathrm{~cm}^{-3}$. For these parameters, the average column density is $N\left(\mathrm{H}_{2}\right)=5.9 \times 10^{21} \mathrm{~cm}^{-2}$ and the corresponding average visual extinction is $A_{\mathrm{V}}=2.8^{\mathrm{m}}$. The cloud is inhomogeneous and the extinction varies depending on the line of sight. In the following, extinction and infrared spectra are only calculated toward one direction. The range of extinctions is $A_{\mathrm{V}}=0.31-24.6$. Higher extinction means larger variations in intensity. In our library method, this should lead to larger errors, at least if the library always contains the same number of intensity intervals.

We created two new models by scaling the densities of the original model. For the radiative transfer problem, the relevant parameter is the column density rather than the volume density and the results of the tests are equally applicable to smaller and correspondingly denser sources. The parameters of the models are listed in Table 1. The visual extinction is in all cases well below $1000^{\mathrm{m}}$ and dust emission can be solved without iteration (see Sect. 2.1).

\subsection{The size of the library}

With discretization reduced to $32^{3}$ cells, the emission spectra can be calculated directly within a reasonable time, and comparison with spectra computed with the method introduced in Sect. 2.2 makes it possible to directly estimate the errors caused by the library method. The radiation field is simulated using the same set of random numbers, and therefore the quoted errors reflect only the accuracy of the approximation. The incoming intensity is estimated with Monte Carlo methods, and the 
resulting random noise causes additional uncertainties. However, although the simulation of the radiation field represents a rather small part of the total computation time in our runs, the expected noise at individual frequencies is only a few per cent. The effect on the dust emission is expected to be somewhat smaller, since the computation of dust temperatures involves averages over a number of frequencies. We computed maps of $32 \times 32$ spectra using the $32^{3}$ cell models and either direct solution or the library method. We first consider models without the PAH component, including only larger carboneous grains and silicate grains.

With relatively low extinction, the model $C 0$ is the easiest case. With two reference wavelengths, $0.12 \mu \mathrm{m}$ and $9.1 \mu \mathrm{m}$, both with 20 intensity intervals, the average relative error in the computed emission was below $2 \%$ in the range $5-300 \mu \mathrm{m}$. Taking into account all spectra and all wavelengths in this range, the maximum deviation between intensities computed directly and with the library method was $\sim 6 \%$. This is very encouraging, considering that in the model the emission per unit volume ( $\sim$ density) varies over nearly three orders of magnitude and the intensity along different lines of sight over almost two orders of magnitude. The variations in the emission per dust grain are also significant, but much smaller, and this makes the use of the library method possible.

The main computational cost lies in the solution of the dust temperature distributions, using the $20 \times 20$ spectra determined by the discretization of the intensity at the two reference wavelengths. Even for such a small model, the computational time is significantly reduced, by a factor of $\sim 30^{3} / 20^{2} \approx 68$.

Figure 2 shows the $100 \mu \mathrm{m}$ emission as the function of the local intensity at two reference frequencies for model $C 0$. In the plot the contours are almost horizontal i.e. the emission can be predicted based on the intensity at only the shorter of the two reference frequencies. In models $C 1$ and $C 2$ the higher extinction makes the second reference wavelength more important. Figure 2 shows clearly that in model $C 2$ the spectrum of the local radiation field can no longer be approximated with only one parameter (e.g. visual extinction), and the isocontours in the figure are not parallel to either axis. At low intensities, i.e. deep inside the cloud, the emission follows the intensity at the longer wavelength and the contours are closer to vertical. At higher intensities (close to the cloud surface) the short wavelength radiation becomes relatively stronger and it is more important in determining the dust temperature and the infrared emission. As a result, the isocontours turn almost horizontal in Fig. 2.

With the previous number of intensity intervals in the library, $20^{2}$, the rms errors increase to $3.5 \%$ for model $C 1$ and $6.2 \%$ for model $C 2$. The errors might be reduced further by optimizing the selection of the reference wavelengths. The wavelengths should obviously be well separated from each other and correspond to sufficiently high optical depths so that intensity variations are well above the Monte Carlo noise. The optimal values depend on the radiation sources, the dust model and the cloud parameters. The reference wavelengths used above are based on some experimentation but are probably not optimal.

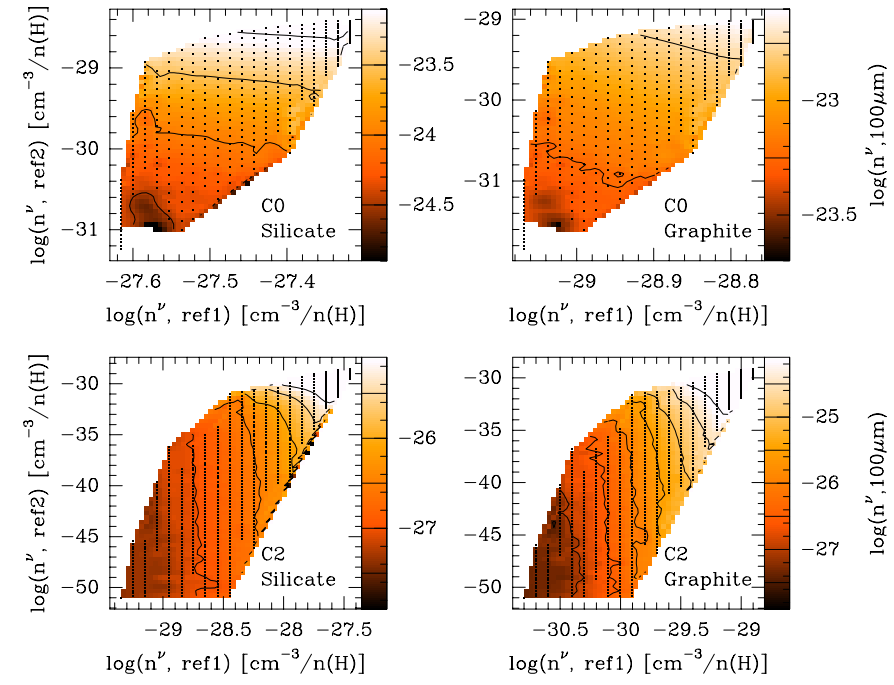

Fig. 2. The $100 \mu \mathrm{m}$ emission as the function of the local radiation field at reference wavelengths, $\lambda_{\text {ref1 }}=9.1 \mu \mathrm{m}$ and $\lambda_{\text {ref2 }}=0.12 \mu \mathrm{m}$. Intensities are given as the number of photons absorbed per cubic centimeter, divided by hydrogen density. The upper frames correspond to model $C 0$ and the lower frames to model $C 2$.

In optically thick clouds, the inclusion of a third reference wavelength may be necessary. It is also better to use longer reference wavelengths in order to avoid the extremely optically thick regime where accurate sampling of the radiation field becomes difficult. Using 20 intensity intervals at wavelengths $0.20,1.7$ and $30 \mu \mathrm{m}$, the rms errors are reduced to $1.8 \%$ and $2.3 \%$ for models $C 0$ and $C 1$, respectively. For the most opaque model, $C 2$, the rms error is $3.8 \%$. It is, however, still possible to increase the size of the library by at least one order of magnitude, and the errors caused by the use of the library method can be kept below the noise due to the Monte Carlo sampling.

The inclusion of PAHs does not cause significant problems for the library method. For the model $C 1$, with a library with three reference wavelengths and $23^{3}$ intensity intervals, the rms error in intensities between $5 \mu \mathrm{m}$ and $300 \mu \mathrm{m}$ becomes $2.3 \%$.

It is not possible to give general estimates of the accuracy of the library method in more complicated situations. For example, the radiation field could probably not be estimated with sufficient accuracy using only two reference wavelengths if the model included two radiation sources with different emission spectra. The local field would clearly depend on the distance to both sources. Furthermore, in an inhomogeneous cloud the way each spectrum is attenuated depends on the density field which modifies both the intensity and the shape of the radiation spectrum. In principle, the accuracy can always be improved by increasing the number of intensity intervals and/or the number of reference wavelengths. The library method remains viable as long as the number of entries in the library is significantly smaller than the number of cells in the cloud. For example, with four reference wavelengths, each with 30 intensity intervals the number library entries is $8.1 \times 10^{5}$ and method could still be used for all models with more that $\sim 100^{3}$ cells. 


\subsection{Effect of cloud resolution on the created library}

When the library is first created, the model cloud is used only to estimate the range of intensities present in the cloud. In the library, the combinations of intensities at the reference wavelengths do not correspond directly to any specific cells in the cloud. Therefore, the previous error estimates should also apply when the same library is used to calculate spectra from models with higher discretization. The only exception may be the coldest spot of the cloud. Low resolution leads to smoothing of density peaks and as a result, a higher resolution model may contain a few cells where the intensity is lower than anywhere in the low resolution model. If the library does not contain entries corresponding to these lowest intensities the spectrum predicted for the coldest core will have a spectrum that is too warm (i.e. the coldest spectrum of the low resolution model).

There are several reasons why the effect is not expected to be very significant. The library represents a mapping between the intensity of the local radiation field and the dust emission per unit density, independent of the density. Comparing models with different discretization, the largest effect on the emitted intensity comes directly from the density. Furthermore, since the radiative transfer equation is integrated over the whole line of sight the relative statistical errors in the emerging intensity are smaller than the relative errors in local emissivity.

We tested the effect explicitly with model $C 1$ discretized into $90^{3}$ cells. The library was built as above (including PAH) but based on simulation of the radiation field in models with either $32^{3}, 64^{3}$ or $90^{3}$ cells. Spectra computed with the different libraries were compared and the results are shown in Table 2. The rms difference between the predicted IRAS intensity ratios, $I_{60} / I_{100}$ are below one percent and only in the coldest core at the position $(-2.1,-1.1)$ the error increases to $\sim 12 \%$. The spectra for this position are shown in Fig. 3. This shows that the spectrum of the coldest spot in the cloud is not necessarily well reproduced when the library is based on a model with crude discretization.

It is not possible to solve the dust temperature distributions for all $90^{3}$ cells in a reasonable time using direct calculations i.e. without the library method. The dust emission was solved, however, for two layers of cells (i.e. two times $90 \times 90$ cells) that included the coldest core identified from the computed map of IRAS ratio $I_{60} / I_{100}$. Altogether $2 \times 90$ spectra were computed for different lines-of-sight along this slice. Comparison of this subset with spectra computed with the library method shows rms difference of $\sim 1-2 \%$, irrespective of the library used. This indicates that, apart from the coldest spot, the libraries are equally good in predicting the infrared spectrum. For the library based on simulations with a $90^{3}$ cloud the maximum deviation at any single frequency is down to $5 \%$.

The effect of the cloud resolution on the created library is relatively small. There are at least two ways to reduce these errors further. One can always create the library using the same discretization as used in the calculation of the spectra. This can be time consuming, but the library method will still be much faster than the direct solutions since the dust emission is solved only for the radiation fields selected in the library and not for all cells. Another possibility is to create the library using a model
Table 2. Comparison of spectra calculated for the model $C 1$. The cloud was discretized into $90^{3}$ cells. The libraries were created using simulations of the radiation field in models with either $32^{3}, 64^{3}$ or $90^{3}$ cells. The last one was used also as the reference to which all other models were compared. The rms difference for range $10-300 \mu \mathrm{m}$ as well as maximum deviation in IRAS ratio $I_{60} / I_{100}$ are shown. The last column shows the comparison with direct calculations (see text).

\begin{tabular}{lllll}
\hline \hline model & library $\left(32^{3}\right)$ & lib. $\left(64^{3}\right)$ & lib. $\left(90^{3}\right)$ & direct \\
\hline $\operatorname{rms}(\Delta I)$ & $0.4 \%$ & $0.15 \%$ & - & $1.3 \%$ \\
$\max \Delta\left(I_{60} / I_{100}\right)$ & $12.4 \%$ & $2.5 \%$ & - & $1.4 \%$ \\
\hline
\end{tabular}

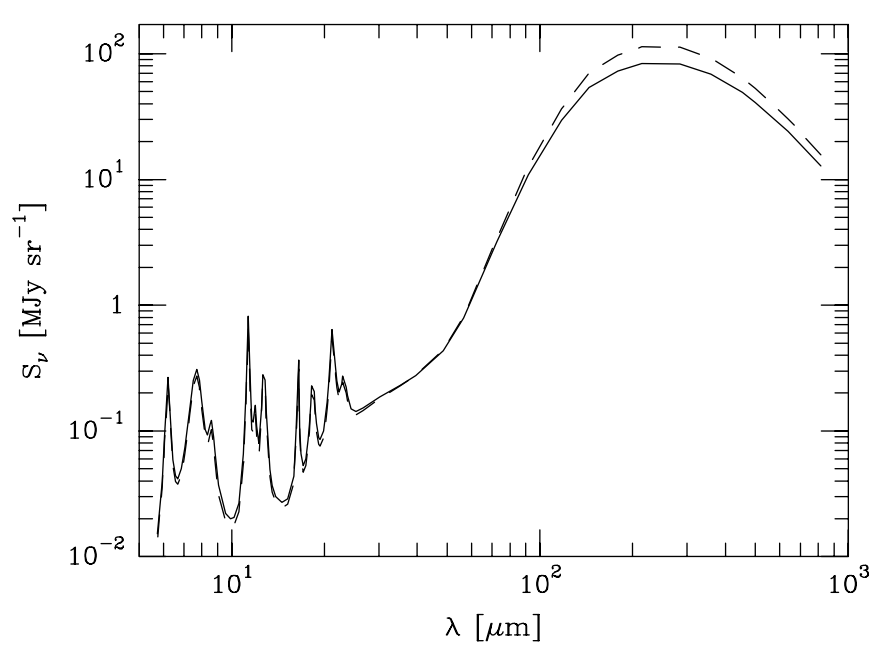

Fig. 3. Spectra calculated for the coldest core in model cloud $C 1$ using the library method. The libraries are based on simulations with models with either $32^{3}$ (dashed line) or $90^{3}$ (solid line) cells. This represents the overall worst case where the library based on $32^{3}$ cell model overestimates e.g. the IRAS ratio $I_{60} / I_{100}$ by more than $10 \%$.

where the range of intensities is artificially increased. This can be achieved by creating the library using a model cloud with a slightly higher density.

\subsection{Effect of cloud resolution on spectra}

In this section we compare spectra calculated for model $C 1$ with cloud discretized into either $32^{3}$ or $128^{3}$ cells. All calculations are made using the same library which includes three reference wavelengths, each with 23 intensity intervals. With increasing resolution, the edges of high density regions are better resolved, and this might lead to changes in the intensity distributions and/or intensity ratios.

Table 3 shows the ranges of surface brightness values for the four IRAS bands for the two discretizations. As expected, with low resolution, the intensity variations are smoothed and the range of intensities is also correspondingly reduced. Observationally, this is more important at the limit of high surface brightness values, where the increase of the spatial resolution by a factor of four increases the peak intensities by approximately the same amount. In the low-resolution model, each spectrum corresponds to an average surface brightness over a larger area, but the values themselves are not significantly biased. For example, the predicted colour ratios shown in Fig. 4 
Table 3. The range of surface brightness values $\left(\mathrm{Jy} \mathrm{sr}^{-1}\right)$ in IRAS bands for models discretized into either $L^{3}=32^{3}$ or $128^{3}$ cells. The power of ten is given in parenthesis.

\begin{tabular}{rllll}
\hline \hline$L$ & $I(12 \mu \mathrm{m})$ & $I(25 \mu \mathrm{m})$ & $I(60 \mu \mathrm{m})$ & $I(100 \mu \mathrm{m})$ \\
\hline 32 & $6.6(4)-1.1(6)$ & $1.5(5)-2.5(6)$ & $4.3(5)-8.5(6)$ & $4.8(6)-7.5(7)$ \\
128 & $5.1(4)-2.0(6)$ & $1.1(5)-3.5(6)$ & $3.4(5)-1.6(7)$ & $3.5(6)-1.4(8)$ \\
\hline
\end{tabular}
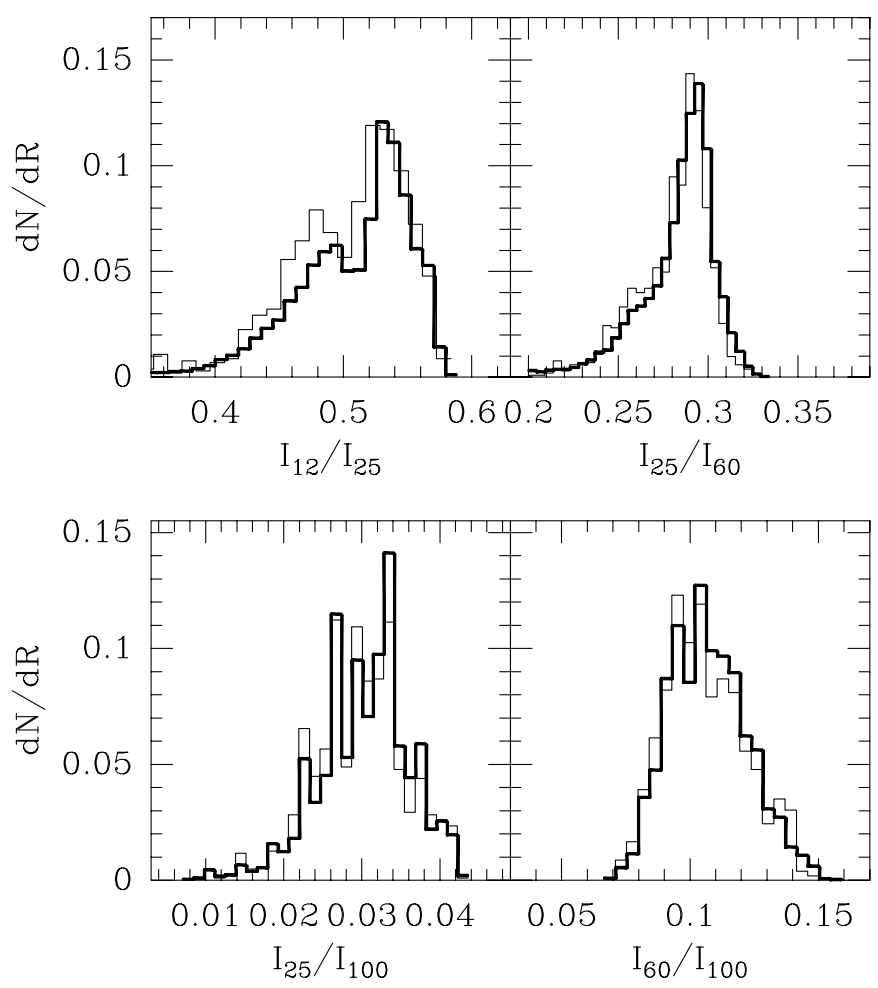

Fig. 4. Distributions of selected IRAS colour ratios in maps computed using either $32^{3}$ (thin line) or $128^{3}$ cell models (thick line).

are essentially the same irrespective of the resolution. The ratio $I_{12} / I_{25}$ changes slightly as the resolution is increased. This ratio traces the PAH component and higher values imply stronger radiation field. In particular, the values in the higher peak at $I_{12} / I_{25} \sim 0.55$ originate mostly from the surface of the projected cloud.

The average properties of the emission, the surface brightness and colour ratios are almost independent of the discretization. Compared with the $32^{3}$ model, however, the calculation with the higher resolution yields an approximately $2 \%$ lower total infrared luminosity. Although small, the change is significant, since the estimated uncertainty per spectrum is already of the same order. The most likely explanation is that at lower resolution, the holes that appear between the filaments are smoothed out, and this increases the total probability that incoming photons are absorbed within the cloud. Bernard et al. (1992) noted that for spherically symmetric models with power law density distributions, the approximation of the radial profile with a step function leads to underestimation of the infrared emission. This effect must also apply to the emission predicted from the cores and filaments in three-dimensional models, especially as the discretization is much more coarse by necessity.
At least in the present case this did not seem to have affected the average surface brightness values. However, without a relatively high resolution, the dense cores would not be resolved at all.

Perhaps the main advantage of high resolution is that it enables statistical studies of the simulated infrared maps. In such studies the map size should preferably be at least $256^{2}$ in order to provide sufficient range of scales.

\section{MHD models}

In this section we present dust emission spectra computed with three-dimensional model $C 1$ consisting of $128^{3}$ cells. Emission is calculated for the range $5 \mu \mathrm{m}-300 \mu \mathrm{m}$ using the dust model of Draine \& Li (2001). The density field is the solution of the three-dimensional MHD equations, in a regime of forced highly supersonic and super-Alfvénic turbulence and with an isothermal equation of state (model $C$ in Juvela et al. 2001). We first present results from the original model and compare them with calculations with lower discretization models. We also discuss the effect of the cloud structure on the observed surface brightness maps and, finally, study qualitatively the effects introduced by spatially varying dust abundances.

\subsection{The original model}

The library (i.e. the mapping between the incoming intensity at selected reference wavelengths and the resulting emission) is created using the original models resampled onto a $32^{3}$ cell grid. We use three reference wavelengths, each with 23 intensity intervals. As described above, the downsized model is needed only to estimate the range of intensities and profiles for the incoming radiation. Therefore, at this stage the spatial resolution is not crucial for the final accuracy. The use of the library leads to significant time saving since in the large, $128^{3}$ cell model the radiative transfer problem is solved only at the three reference wavelengths. In this way the computational time is reduced by a factor of $\sim 100$ (ratio of total number of simulated wavelengths and number of reference wavelengths). The main cost lies, however, in the solving of the dust temperature distributions. Here the time saving for the $128^{3}$ cloud is a factor of $128^{3} / 23^{3} \sim 170$. As shown in Sect. 3 , the expected rms error in the computed spectra is less than $2 \%$.

The surface brightness maps for the four IRAS bands are shown in Fig. 5. The maps have been calculated using the bandpass profiles given in the IRAS Explanatory Supplement (Beichmann et al. 1998). In all four maps the surface brightness probes the same filamentary density field. Maps of surface brightness ratios (Fig. 6) show, however, clear variations in the FIR colours and, as expected, dense cores and filaments are clearly visible through their lower colour temperatures.

Figure 7 shows the average infrared spectrum and two individual spectra with extreme values of the ratio between $15 \mu \mathrm{m}$ and $600 \mu \mathrm{m}$ intensities. The spectrum with relatively strongest mid-infrared emission originates from the upper left hand corner i.e. gas subjected almost to the full intensity of the external radiation field. The coldest spectrum is observed at the core located at $(-2.1,-1.1)$. There the emission peaks close to 
$\gtrsim 200 \mu \mathrm{m}$ but the core is already quite noticeable in the maps of IRAS colour ratios (Fig. 6).

For the Li \& Draine (2001) dust model, the ratio $I_{60} / I_{100}$ is close to 0.2 when dust is exposed to the pure interstellar radiation field. The dust model of Désert et al. (1990) predicts the same ratio $I_{60} / I_{100} \sim 0.2$. This is, of course, not surprising since both dust models are created to the reproduce existing IRAS and COBE measurements. The ratio of 0.2 is similar to those found towards many diffuse clouds (e.g. Laureijs et al. 1991). This ratio applies, however, only to the diffuse medium. In our cloud $C 1$, the average ratio is $\sim 0.11$. This cloud is quite opaque, with mean visual extinction of $A_{\mathrm{V}} \sim 11$. The model $C 0$ has lower visual extinction, $\left\langle A_{\mathrm{V}}\right\rangle \sim 2.8$, and is closer to typical regions of molecular clouds. In $C 0$, the average colour ratio is $\left\langle I_{60} / I_{100}\right\rangle \sim 0.15$. This is the same as the value found by Abergel et al. (1994) for the Taurus molecular cloud complex.

Some of the lowest colour temperatures are reached in the filament in the upper right hand corner of the maps. Like many other features, this is actually a superposition of separate filaments or sheet-like structures along the line of sight. In this direction, the densest filament is $\sim 1 \mathrm{pc}$ in diameter and has a maximum density of $\gtrsim 10^{4} \mathrm{~cm}^{-3}$. This roughly corresponds to a visual extinction of $10^{\mathrm{m}}$ across the filament $\left(\sim 5^{\mathrm{m}}\right.$ to the centre of the filament). There is, however, significant additional extinction from the more diffuse material between the dense regions. The ratio $I_{60} / I_{100}$ decreases within the filament by a factor of $\sim 2.0$ relative to the average value over the map, and is therefore approximately 4 times lower than the value given by the dust model in the diffuse medium. In observations the ratio is often found to vary by a larger amount. In Lynds 134 the ratio decreases by a factor of $\sim 7$ from the diffuse medium to the cloud cores, where the visual extinction is $A_{\mathrm{V}} \sim 10$, close to that in our model $C 1$ (Laureijs et al.1991). In the cirrus cloud MCLD 123.5+24.9 in the Polaris Flare the observed ratio $I_{60} / I_{100}$ is 0.034 (Bernard et al. 1999) while the quoted average value for cirrus clouds is $\left\langle I_{60} / I_{100}\right\rangle \sim 0.31$ ) (Lagache et al. 1998). The ratio $\left\langle I_{60} / I_{100}\right\rangle$ has therefore decreased by a factor of $\sim 9$, although the extinction is lower than in the filament in our model. As discussed by Bernard et al. (1999), the explanation of the observations requires spatial variation of the dust properties. We will study these effects qualitatively in Sect. 4.3. The calculations were carried out using a cloud divided into $128^{3}$ cells while the library was created with a model with only $32^{3}$ cells. However, according to Sect. 3 the expected rms error of the spectra is only $\sim 2 \%$ and the error in $I_{60} / I_{100}$ is less than $15 \%$.

The average ratio $\left\langle I_{140} / I_{240}\right\rangle$ calculated using the DIRBE system response functions is $\sim 0.88$ for model $C 1$ and $\sim 1.05$ for model $C 0$. In the spherically symmetric models of Bernard et al. (1992; see Lagache et al.1998, Table 3), similar ratios are obtained only when the extinction is low compared with the average extinction in our models. For example, with $A_{\mathrm{V}}=4$ towards the cloud centre, their ratio has already decreased to 0.77 , well below the value in model $C 1$ which has, nevertheless, higher average visual extinction. This may be partly caused by differences in the dust models, but it is mainly the result of the inhomogeneity of our models. In an inhomogeneous cloud, the ratio remains higher because there are no large,

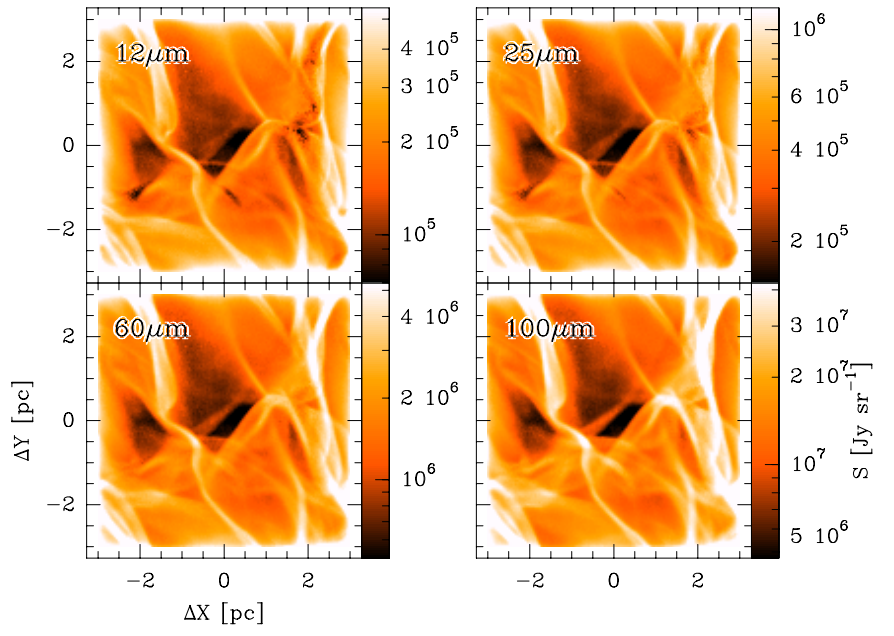

Fig. 5. Surface brightness in the four IRAS bands computed for the model $C 1$.

connected regions completely shielded from the external radiation field.

The spatial power spectra for the $12 \mu \mathrm{m}$ and $100 \mu \mathrm{m}$ maps are shown in Fig. 8. These are one-dimensional power spectra, i.e. they are computed from the corresponding two-dimensional power spectra by averaging over annuli of constant wavenumber. Although the MHD simulation used periodic boundary conditions, the external heating introduces radial gradients in the infrared maps, and therefore direct comparison with observations is not possible at the largest scales. We will address this question in future work, where more detailed modelling will be done, also taking into account the asymmetry of the galactic radiation field.

The finite spatial resolution of the MHD simulation and the relatively small size of the maps, $128 \times 128$ pixels, further limits the available dynamic range. In Fig. 8 the vertical lines show the range where the spectrum follows an approximate power law, and where we determine the exponent of the power law. We obtain exponents $\beta=-2.54$ and -2.72 for $12 \mu \mathrm{m}$ and $100 \mu \mathrm{m}$, respectively. In Sect. 3 we concluded the accuracy of the computed spectra is probably of the order of $2 \%$ per frequency point. Averaged over the IRAS filters the noise should be much lower. This is confirmed by the fact that the power spectra do not show a flat part typical of noise. Assigning an uncertainty of $2 \%$ to the computed IRAS values we estimated with Monte Carlo methods an uncertainty of $\sim 0.01$ for the exponent of the power law. This error estimate is valid for the comparison of maps with the same underlying density distribution. The exponent could be changed significantly more by selecting a different range of spatial frequencies or e.g. a different projection of the cloud.

The power law exponent for the column density map is -2.80 . The same power spectrum slope is predicted analytically for the projected density in supersonic turbulence by Boldyrev et al. (2002), based on the velocity structure function scaling predicted by Boldyrev (2002).

Compared with the column density maps the infrared maps have more power on small scales but the slopes are in the range obtained from observations. The power law exponent of IRAS 

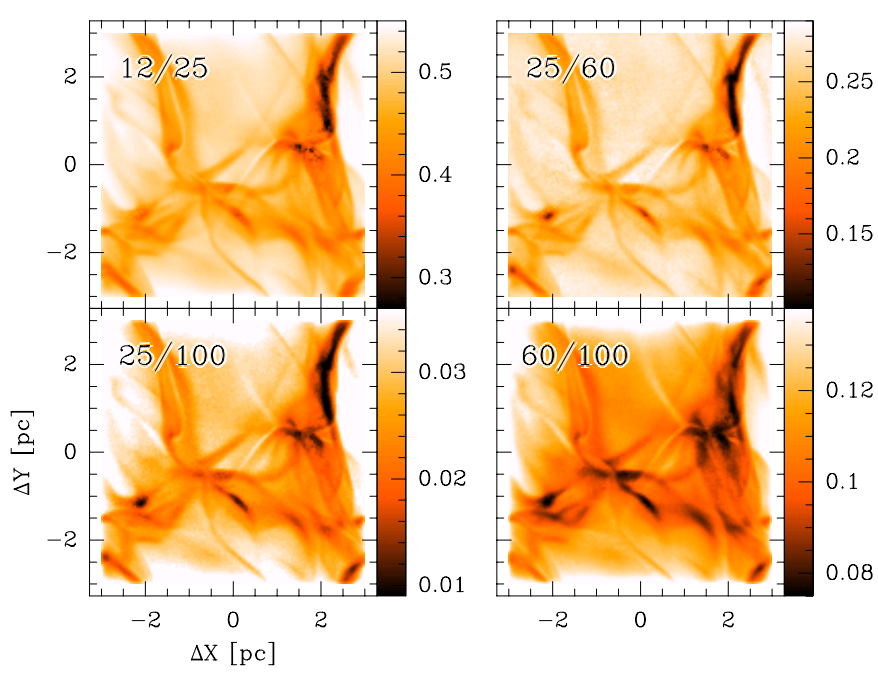

Fig. 6. Ratios between surface brightness values in IRAS bands.

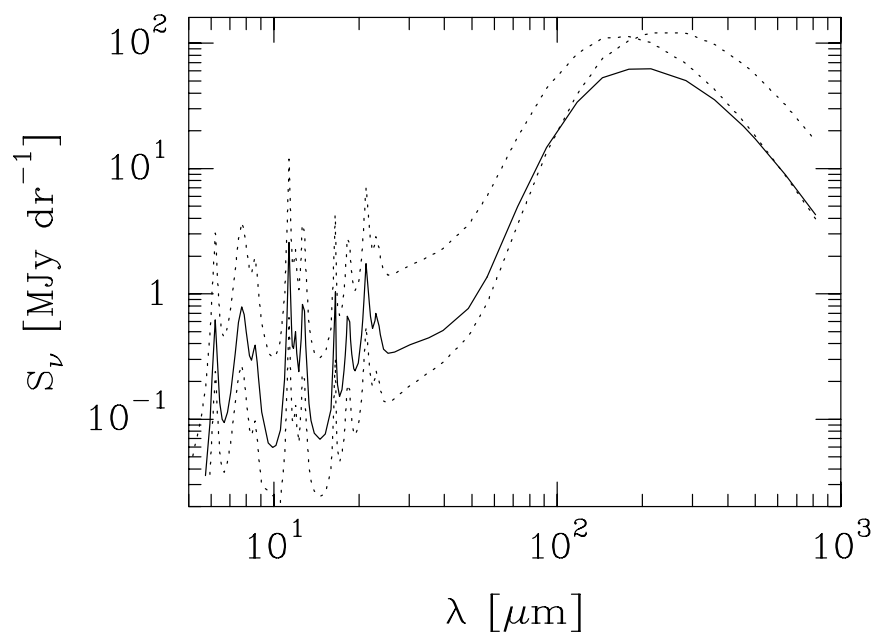

Fig. 7. Average infrared spectrum observed from model $C 1$. The dotted lines show two individual spectra with extreme ratios between $15 \mu \mathrm{m}$ and $600 \mu \mathrm{m}$ intensities.

maps determined by Gautier et al. (1992) was $k=-3.0$. Based on ISO observations, however, Herbstmeier et al. (1998) found different power laws in different clouds, from very shallow spectra, $k \sim-1$, in dark regions up to $k=-3.6$ in the Cepheus flare.

The power law exponent of the infrared maps is mostly a result of the underlying density distribution. The infrared maps do contain, however, significantly more power at high spatial frequencies, above the range used to compute the values of the power law exponents. This is due to dust temperature variations that cause intensity fluctuations on top of the column density distribution. The $12 \mu \mathrm{m}$ map seems to have more small scale structure than the $100 \mu \mathrm{m}$ one. At these high frequencies the underlying density distribution is affected by the finite resolution of the MHD simulations. However, for the given density distribution we can compare the power spectra up to the Nyquist limit i.e. in Fig. 8 up to $\sim 10 \mathrm{kpc}^{-1}$. The differences were also observed in other models and they are well above the Poisson noise from the Monte Carlo simulation. Most of the fluctuations in the power spectra are caused by the underlying, common density distribution and e.g. the difference $12 \mu \mathrm{m}$ and $100 \mu \mathrm{m}$ is more significant than a comparison with the apparent noise in Fig. 8 would lead to believe. The quantitative result could, however, change with better spatial resolution if the density field contained significant small scales structures.

At $12 \mu \mathrm{m}$, the emission mostly depends on the heating by short wavelength photons that are absorbed at the surface of the density structures. On the other hand, the heating of the large grains varies less rapidly, and one could expect the $100 \mu \mathrm{m}$ map to show relatively less small scale structures. Nevertheless, analysis of IRAS maps has not shown clear differences between the different bands. It is conceivable that in the observations, the differences are masked by projection effects or by the spatial variation in dust abundances and properties.

\subsection{Effects of inhomogeneity}

To check to what extent the three-dimensional cloud structure affects the properties of infrared emission, we compute spectra from two modified models, one with a smoother density structure and one with a smaller density contrast than model $C 1$.

In the case of the first model, $C 1 a$, we re-arrange the cells in model $C 1$ so that in each column along the line of sight, the density increases monotonically towards a plane that is perpendicular to the line-of-sight and goes through the cloud centre. In this way, both the column density field and the probability density function of the volume density are the same as in the original model $\mathrm{C} 1$, but the density structure is smoother. Comparison with the original model showed some noticeable changes in the surface brightness distribution, which now follows more closely the column density distribution. In the original cloud, the observed infrared intensities are more sensitive to the intensity of the radiation field at the location of each individual filament or core. For example, the filament going through position $(-2,2)$ is brighter in Fig. 5 than in Fig. 9 because it is located near the surface of the cloud.

In model $C 1 a$, there are also some changes in the distribution of IRAS colours (see Fig. 9). There is a tighter correlation between $60 \mu \mathrm{m}$ and $100 \mu \mathrm{m}$ intensities than in the original model $C 1$. This is expected, as the cloud is inhomogeneous (clumpy or filamentary) essentially only in the plane of the sky, and smooth in the third spatial dimension.

The modified cloud $C 1$ is not "clumpy" along the line of sight, and following the preceding discussion on the effects of resolution, one might expect higher infrared emission. However, the emission is some $20 \%$ lower. This is a direct consequence of the fact that the modified cloud is effectively smaller along the line of sight. Since most of the mass is concentrated in a smaller volume, less radiation enters dense parts of the cloud and the total absorbed energy is also correspondingly lower. Conversely, if dust properties and the external radiation field were known to an accuracy of a few percent, it would be possible to make rough estimates of the line-of-sight size of clouds, even when the degree of clumpiness in this direction is not known. 


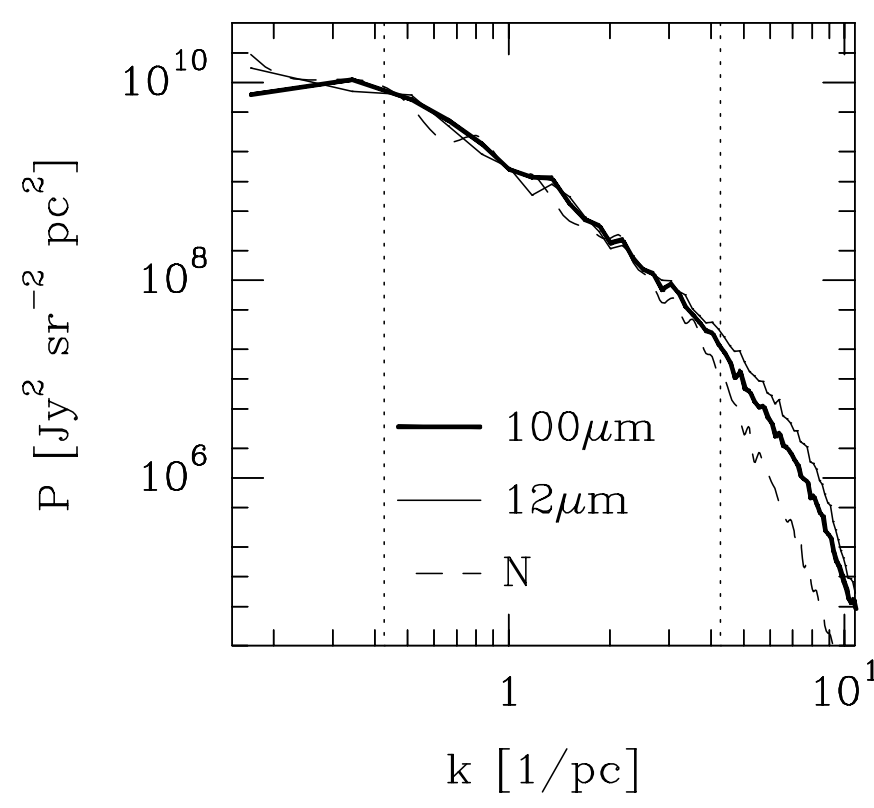

Fig. 8. Spatial power spectra of the $12 \mu \mathrm{m}$ (thin line) and $100 \mu \mathrm{m}$ (thick line) surface brightness maps computed from model $C 1$. The $100 \mu \mathrm{m}$ curve has been scaled down by a factor of 5000. The Nyquist limit corresponds to $k=10.7 \mathrm{pc}^{-1}$, but since the actual resolution of the MHD simulation is somewhat lower, the power already drops above $k \sim 5 \mathrm{pc}^{-1}$. The dashed curve shows the power spectrum of the underlying column density map with arbitrary scaling. The vertical lines limit the range used to compute the exponents of the corresponding power laws.

In model $C 1$, the ratio between the highest and lowest densities is close to $6 \times 10^{4}$. We produced another, more uniform cloud model, $C 1 b$. By applying a linear scaling to the density values, the total density contrast was reduced by a factor of 100 . The average column density remains the same as in models $C 1$ and $C 1 a$, but the absorbed energy is higher since it depends on column density via an exponential function. As a result, compared with the original model $C 1$, the total emitted energy increases by $\sim 7 \%$. For the IRAS bands, the difference is slightly higher, $\sim 8.5 \%$. In spite of the increased energy input, the average ratio between IRAS bands gives a lower colour temperature. The difference is probably real, although it is only a couple of per cent, which is close to the expected accuracy of the calculations. In model $C 1 b$, near- and mid-infrared emission is concentrated on the surface of the model cloud, and the inner parts are correspondingly cooler. The infrared colours were averaged over all spectra without weighting them with the corresponding intensities. Consequently, the average colours can indicate lower dust temperatures than in the model $C 1$, where the short wavelength emission is spread more evenly over the projected cloud area.

The power spectra of the $12 \mu \mathrm{m}$ and $100 \mu \mathrm{m}$ maps are almost identical in the range (see Fig. 8) used for the determination of the power law exponent and the exponent is $\sim-2.7$ for both maps. If the power spectra are scaled on top of each other in this range of spatial frequencies, the $12 \mu \mathrm{m}$ curve is only slightly above the $100 \mu \mathrm{m}$ curve at high frequencies, while at
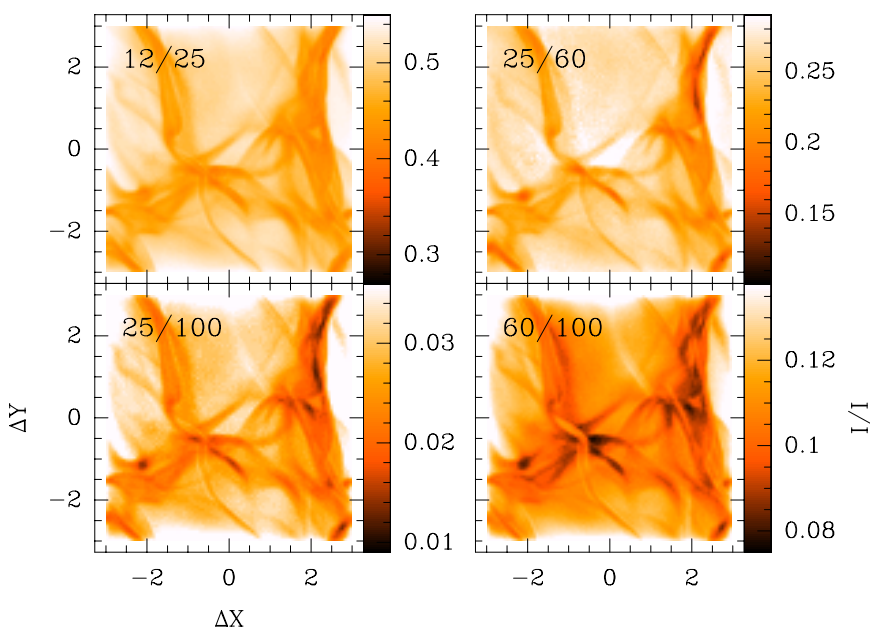

Fig. 9. IRAS colours for model $C 1 a$, a modification of model $C 1$ where the density structure along the line of sight has been made smooth by re-arranging the cells along the line of sight (see text).

low frequencies, the $12 \mu \mathrm{m}$ is significantly higher, due to the stronger brightening towards the cloud surface.

\subsection{Dust abundance variations}

There is strong observational evidence that the dust properties change, not only in dense cloud cores (e.g. Laureijs et al. 1996) but also in clouds with moderate visual extinction (Bernard et al. 1999; Cambrésy et al. 2001; Stepnik et al. 2001; Miville-Deschênes et al. 2002). Possible causes are the growth of grain mantles and the formation of large dust aggregates. The processes can take place only in dense medium that is protected from the external UV radiation field.

We use the three-dimensional model $C 1$ to study the qualitative effects of abundance changes on the observed infrared radiation. The model has mean density $\sim 10^{3} \mathrm{~cm}^{-3}$ with peak values higher by two orders of magnitude.

We adjust the abundances of the grain populations according to the local density only. The modified model is called $C 1 c$. At each position, the dust is composed of five components, the first three of which are $\mathrm{PAH}$, silicate and carbon grains of the Li \& Draine model (2001). The additional two components are modified silicate and carbon grain populations with a flatter size distribution: the original exponent of the size distribution is adjusted so that the original number ratio of grains below and above $25 \mathrm{~nm}$ is reduced by $50 \%$. At low densities, the abundance of the new components is zero. Their abundance increases linearly with the logarithm of the density. The abundance of the unmodified grain populations is decreased linearly with respect to the logarithm of density and the total mass of dust remains unchanged. Above $10^{4} \mathrm{~cm}^{-3}$, all dust is in the modified silicate and carbon grain populations. This is, of course, only a qualitative description of the expected dust abundance variations. In particular, we ignore all changes in the dust optical properties expected to be caused by the formation of aggregate grains. As a result, the model can be expected to underestimate true colour temperature variations across dense filaments or cores (see e.g. Stepnik et al. 2001). 

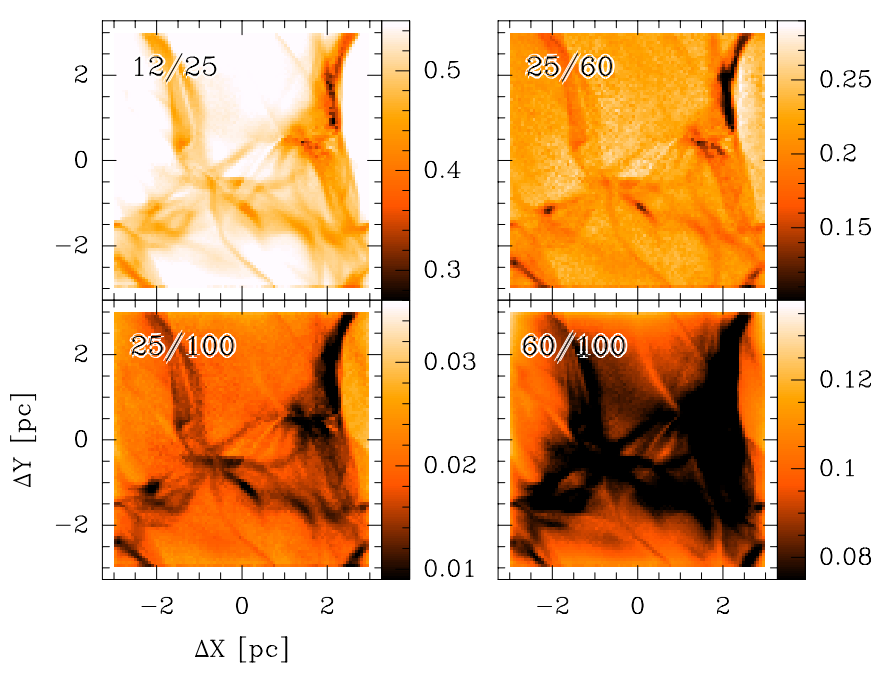

Fig. 10. Maps of selected IRAS colours calculated for the model $C 1 c$. In the model the dust abundances vary depending on the local density (see text). The colour scales are the same as in Fig. 6.

Figure 10 shows maps of IRAS intensity ratios in model $C 1 c$. Since we have reduced the number of small grains the average colour temperatures have dropped. The ratio $\left\langle I_{25} / I_{100}\right\rangle$ has decreased the most, by $34 \%$. The ratio $\left\langle I_{60} / I_{100}\right\rangle$ is now lower by $23 \%$. The average $100 \mu \mathrm{m}$ surface brightness has only increased by $\sim 17 \%$ and the $60 \mu \mathrm{m}$ emission is below that of model $C 1$.

The $12 \mu \mathrm{m}$ map contains relatively more power at small scales. The short wavelength maps are not expected to change much, since most of the emission comes from low density material where grain abundances remain almost unchanged. Abundance variations should increase far-infrared emission in the densest regions, and the corresponding maps are, therefore, expected to contain more power at high spatial frequencies. However, the changes in the power spectra remain very small. The new exponents of the power laws are -2.51 and -2.67 for $12 \mu \mathrm{m}$ and $100 \mu \mathrm{m}$, respectively; the $100 \mu \mathrm{m}$ slope has only flattened by $\sim 2 \%$ relative to the original model $C 1$.

In the filament at position $(2,2)$ the $12 \mu \mathrm{m}$ surface brightness has decreased by $\sim 30 \%$, while the $100 \mu \mathrm{m}$ emission has increased less than 10\%: this is less than the average increase over the whole map. The change in the dust abundances leads to lower extinction, especially at short wavelengths, and this decreases temperature variations across the filament. The lowest values of the ratio $I_{60} / I_{100}$ are $\sim 0.05$ in this region, i.e. some $30 \%$ lower than in the original cloud $C 1$. Compared with the diffuse medium value of 0.2 , the reduction in $I_{60} / I_{100}$ is still only a factor $\sim 4$. This is still less than found in many observations (e.g. Laureijs et al. 1991; Bernard et al. 1999). The model cloud has very significant extinction, even outside the densest filaments, and this should help to further decrease the $I_{60} / I_{100}$ ratio.

In the presented model, we have only adjusted the grain size distributions by halving the ratio of grains below and above $25 \mathrm{~nm}$ in dense cloud regions with $n>10^{4} \mathrm{~cm}^{-3}$. There is some evidence that dust properties can undergo significant changes even at much lower densities, below $10^{3} \mathrm{~cm}^{-3}$ (e.g. Bernard et al. 1999). On the other hand, sub-mm observations of dense cores seem to indicate that in those environments, all small grains may be removed (e.g. Stepnik et al. 2001). If small dust grains stick onto the surfaces of larger grains, the optical properties of the grains will change and lead to a lower physical temperature. As a result, the farinfrared colour temperatures decrease, and this can explain the very low $I_{60} / I_{100}$-ratios observed. In dense environments, dust may also form larger, fluffy aggregate particles that have similarly enhanced far-infrared emissivity and low physical temperature (Mathis \& Whiffen 1989; Ossenkopf 1993; Wright 1987; Bazell \& Dwek 1990). Many of the observed cold cores are too small for the low colour temperatures to be explained merely by the attenuation of the external radiation field. The need for large variations in the abundance ratio of large and small dust grains is, therefore, already well established.

\section{Conclusions}

We have presented a new approximate method that can be used to compute the infrared dust emission from large, three dimensional cloud models consisting of millions of computational cells. The method is based on simple discretization of the incoming intensity using a few reference wavelengths. We have demonstrated that relative accuracy of a few percent is easily reached, even when transiently heated grains are included. Such accuracy is hard to match either by observations or the Monte Carlo sampling that is often used to estimate the intensity of the radiation field. We have tested the method using model clouds with average visual extinctions up to $A_{\mathrm{V}} \sim 100$. However, with use of more reference wavelengths and/or higher discretization, the method can be applied to even denser clouds, and to models with several heating sources with different emission spectra.

We have used our new approximate method to compute dust emission, from near-infrared to sub-mm wavelengths, from models based on MHD simulations with supersonic and super-Alfvénic turbulence. The spatial discretization was found to have little impact on the derived distributions of the intensity ratios between IRAS bands. On the other hand, spatial averaging will always reduce the range of intensities. High resolution calculations are therefore necessary, especially when the computed infrared maps are used for statistical studies or for direct comparison with observations.

The spatial power spectra at different wavelengths generally show little variation. The shorter wavelengths have, however, slightly higher relative power at higher spatial frequencies. For the selected MHD model, the power spectra follow approximately a power law with an exponent between -2.5 and -2.7 , while the power spectrum of the underlying column density map has a slope of -2.8 .

We qualitatively studied the effect of density dependent dust size distributions on the observable infrared maps. By reducing the relative abundance of very small grains by $50 \%$ in regions with densities $n>10^{4} \mathrm{~cm}^{-3}$, the ratio $I_{60} / I_{100}$ could be brought down to $\sim 0.05$ in dense regions where visual extinction is $A_{\mathrm{V}} \sim 10$. In observations, the colour ratio can reach lower values even in less opaque clouds. This indicates that 
dust properties must undergo even larger changes, with the removal of most of the small grains and the growth of larger ones.

The numerical method presented in this work will be very useful to constrain dust properties by comparison with new observational data. Dust column density maps of large molecular cloud complexes can now be obtained from stellar extinction measurements based on the "Two Microns All Sky Survey" (2MASS). Padoan et al. (2002) have recently generated extinction maps of the whole Taurus molecular clouds complex using 2MASS data, with a spatial resolution comparable or better than that of $100 \mu \mathrm{m}$ IRAS images, spanning a range of visual extinction from $A_{\mathrm{V}} \approx 0.3 \mathrm{mag}$ to $A_{\mathrm{V}} \approx 30 \mathrm{mag}$. The comparison of this type of dust column density maps with IRAS images and, in the near future, with SIRTF-MIPS images will provide new observational constraints over a large range of column density and gas density values. Detailed three dimensional calculations of dust emission from physical models of interstellar clouds will be necessary for reproducing the observational data and improve our knowledge of the properties of interstellar dust.

Acknowledgements. We thank the referee, Dr. J. Le Bourlot, for comments that have improved the paper. M.J. acknowledges the support of the Academy of Finland Grants no. 1011055, 166322, 175068, 174854. The work of P.P. was performed while P.P. held a National Research Council Associateship Award at the Jet Propulsion Laboratory, California Institute of Technology.

\section{References}

Abergel, A., Boulanger, F., Mizuno, A., \& Fukui, Y. 1994, ApJ, 423, L59

Bazell, D., \& Dwek, E. 1990, ApJ, 360, 142

Beichman, C. A., Neugebauer, G., Habing, H. J., et al. 1998, IRAS Explanatory Supplement

Bernard, J. P., Boulanger, F., Desert, F. X., \& Puget, J. L. 1992, A\&A, 263,258
Bernard, J.-P., Abergel, A., Ristorcelli, I., et al. 1999, A\&A, 347, 640 Boldyrev, S., Nordlund, Å., \& Padoan, P. 2002, Phys. Rev. E, in press Boldyrev, S. 2002, ApJ, 569, 841

Boulanger, F., Abergel, A., Bernard, J. P., et al. 1996, A\&A, 312, 256

Cambrésy, L., Boulanger, F., Lagache, G., \& Stepnik, B. 2001, A\&A, 375, 999

Désert, F.-X., Boulanger, F., \& Puget, J. L. 1990, A\&A, 237, 215

Draine, B. T., \& Li, A. 2001, ApJ, 551, 807

Dwek, E., Arendt, R. G., Fixsen, D. J., et al. 1997, ApJ, 475, 565

Finkbeiner, D. P., Davis, M., \& Schlegel, D. J. 1999, ApJ, 524, 867

Gautier, C., III, Boulanger, F., Perault, M., \& Puget, J. L. 1992, AJ, 103,1313

Henyey, L. G., \& Greenstein, J. L. 1941, ApJ, 93, 70

Herbstmeier, U., Abraham, P., Lemke, D., et al. 1998, A\&A, 332, 739

Hollenbach, D. J., Takahashi, T., \& Tielens, A. 1991, ApJ, 377, 192

Juvela, M. 1997, A\&A, 322, 943

Juvela, M. 1998, A\&A, 329, 659

Juvela, M., Padoan, P., \& Nordlund, A. 2001, ApJ, 563, 853

Juvela, M., Mattila, K., Lehtinen, K., et al. 2002, A\&A, 382, 583

Krügel, E., \& Walmsley, C. M. 1984, A\&A, 130, 5

Lagache, G., Abergel, A., Boulanger, F., \& Puget, J.-L. 1998, A\&A, 333, 709

Laor, A., \& Draine, B. T. 1993, ApJ, 402, 441

Laureijs, R. J., Clark, F. O., \& Prusti, T. 1991, ApJ, 372, 185

Laureijs, R. J., Haikala, L., Burgdorf, M., et al. 1996, A\&A, 315, L317

Léger, A., d'Hendecourt, L., \& Défourneau, D. 1989, A\&A, 216, 148

Li, A., \& Draine, B. T. 2001, ApJ, 554, 778

Mathis, J. S., Mezger, P. G., \& Panagia, N. 1983, A\&A, 128, 212

Mathis, J. S., \& Whiffen, G. 1989, ApJ, 341, 808

Miville-Deschênes, M.-A., Boulanger, F., Joncas, G., \& Falgarone, E. 2002, A\&A, 381, 209

Ossenkopf, V. 1993, ApJ, 280, 617

Ossenkopf, V. 2002, A\&A, 391, 295

Padoan, P., Juvela, M., Bally, J., \& Nordlund, Å. 1998, ApJ, 504, 300

Padoan, P., Cambrésy, L., \& Langer, W. 2002, ApJ, submitted [astro-ph/0208217]

Park, Y.-S., \& Hong, S. S. 1995, A\&A, 300, 890

Park, Y.-S., Hong, S. S., \& Minh, Y. C., 1996, A\&A, 312, 981

Stepnik, B., Abergel, A., Bernard, J.-P., et al. 2001, ESA-SP, 460, 269

Wright, E. L. 1987, ApJ, 320, 818 